\title{
Female Fertility Following Chemotherapy for Breast Cancer: A Descriptive Study of 265 Cases Treated in Yaounde
}

\author{
Félix Essiben ${ }^{*}$, Ngowa Jean Dupont Kemfang1, Aline Nganso ${ }^{2}$, Meka Juliette Esther Ngo Um ${ }^{3}$, \\ Etienne Atenguena ${ }^{4}$, Samuel Ojong ${ }^{2}$, Paul Ndom ${ }^{5}$, Emile Telesphore Mboudou 6
}

\author{
${ }^{1}$ Yaoundé Central Hospital, Department of Gynecology and Obstetrics, Faculty of Medicine and Biomedical Sciences, University \\ of Yaoundé I, Yaoundé, Cameroon \\ ${ }^{2}$ Department of Gynecology and Obstetrics, Faculty of Medicine and Biomedical Sciences, University of Yaoundé I, Yaoundé, \\ Cameroon \\ ${ }^{3}$ Yaounde Gyneco-Obstetric and Pediatric Hospital, Department of Gynecology and Obstetrics, Faculty of Medicine and \\ Biomedical Sciences, University of Yaoundé I, Yaoundé, Cameroon \\ ${ }^{4}$ Yaounde General Hospital, Yaoundé, Cameroon \\ ${ }^{5}$ Yaoundé General Hospital, Department of Internal Medicine and Specialities, Faculty of Medicine and Biomedical Sciences, \\ University of Yaoundé I, Yaoundé, Cameroon \\ ${ }^{6}$ Douala Gyneco-Obstetric and Pediatric Hospital, Department of Gynecology and Obstetrics, Faculty of Medicine and \\ Biomedical Sciences, University of Yaoundé I, Yaoundé, Cameroon \\ Email: *essibenx@yahoo.com, jdkemfang@yahoo.fr, lilydjokou@yahoo.com, estherum@yahoo.fr, \\ atenguenatien2002@gmail.com, ojongsamuel27@gmail.com,ndompaul@yahoo.fr, mboudoudoc@yahoo.fr
}

\begin{abstract}
How to cite this paper: Essiben, F., Kemfang, N.J.D., Nganso, A., Ngo Um, M.J.E., Atenguena, E., Ojong, S., Ndom, P. and Mboudou, E.T. (2020) Female Fertility Following Chemotherapy for Breast Cancer: A Descriptive Study of 265 Cases Treated in Yaounde. Open Journal of Obstetrics and Gynecology, 10, 166-178. https://doi.org/10.4236/ojog.2020.1010014
\end{abstract}

Received: November 11, 2019

Accepted: January 10, 2020

Published: January 13, 2020

Copyright ( 2020 by author(s) and Scientific Research Publishing Inc. This work is licensed under the Creative Commons Attribution International License (CC BY 4.0).

http://creativecommons.org/licenses/by/4.0/

c) (i) Open Access

\begin{abstract}
Context: In Cameroon, breast cancer (BC) is usually diagnosed late in the disease course. About a third of women affected are aged less than 40 years. Chemotherapy (CT) could alter ovarian function and thereby compromise future fertility in these women. We therefore described the fertility of women following CT for BC in women treated at the Yaounde General Hospital (YGH). Methods: It was a retrospective, descriptive, cross-sectional study conducted over 8 months, from January to August 2017. We used the files of patients managed for BC from January 2011 to December 2015 in the medical oncology unit of the YGH. Results: We included 265 patients for the study following at least one year of CT. The mean age at the onset of CT was $35.9 \pm$ 6.9 years. Most of the patients had fewer than 2 deliveries (68.3\%). Intraductal carcinoma was the most frequent histological lesion (90.6\%) and most often diagnosed in advanced stages $(73.5 \%)$. As per CT, the FAC protocol was the most used (44.5\%). The menstrual cycles of the patients were more regular prior to the onset of chemotherapy $(89.4 \%$ vs. $58.9 \%)$, while the mean period of resumption of menstrual activity following cessation of chemotherapy was $6.3 \pm 2.0$ months. Chemotherapy-related amenorrhoea was observed in $29.1 \%$ of patients after 12 months of treatment. More than half of these women
\end{abstract}


(51.3\%) complained of a drop in libido and 6.4\% achieved pregnancy within 13 months following cessation of chemotherapy. Conclusion: Following chemotherapy for $\mathrm{BC}$, menstrual cycle disorders are more frequent and this can affect the patients' reproductive potential. Fertility consultations should be integrated into the management plan of such patients.

\section{Keywords}

Chemotherapy, Fertility, Women of Childbearing Age, Breast Cancer, Yaounde

\section{Introduction}

Breast cancer is the first female cancer in Cameroon [1], with a significant number of young women amongst those affected [2] [3]. Many studies suggest the occurrence of more aggressive forms of breast cancer in young women [4] [5] [6]. The absence of screening in young people, associated to the low levels of knowledge on the condition is responsible for the late diagnosis of breast cancer [3] [7]. As a result, practitioners resort to more aggressive treatment protocols to ensure survival and/or remission.

In addition to its aesthetic and psychological complications, the management of breast cancer in women of childbearing age could decrease their fertility potential. Because of their young ages, advanced disease, and the aggressive nature of the tumours, chemotherapy constitutes the mainstay of conservative management plans. According to Rudra et al. in the United States, a higher proportion of women under 40 years of age require chemotherapy when compared to older women [8]. Chemotherapy is based on antimitotic regimens with well described effects on ovarian function. These drugs act through multiple mechanisms such as decreased blood flow to the ovaries, the destruction of the ovarian cortical vasculature with ensuing cortical fibrosis associated to the disappearance of primordial follicles [9] [10]. Chemotherapy accelerates physiological ovarian aging, and therefore significantly compromises the fertility of women.

Improvements in treatment options and a better codification of the management of breast cancers have significantly increased the survival of patients. There is a need to address the desire for childbearing in women after breast cancer treatment, because the recovery of ovarian function and fertility becomes an important goal for their post-treatment quality and hence the need to perform fertility assessments while managing young women for breast cancer [11].

In this study, we aimed to describe the characteristics of reproductive life as well as the fertility of women following treatment for breast cancer with chemotherapy. Alterations in ovarian function following chemotherapy, raise the need to resort to the increasingly available and accessible gamete conservation techniques.

\section{Materials and Methods}

We conducted a descriptive cross-sectional study of a population of women of 
childbearing age, treated for breast cancer at the medical Oncology unit of the Yaoundé General Hospital over a period of 5 years from January 2011 to December 2015. The study took place from January 2017 to August 2017 (8 months). We collected the contact details of the patients followed up for breast cancer from the database of the medical oncology unit. Then, using these, we searched for and consulted their medical files at the hospital archives. The medical records granted us access to the patients' telephone numbers via which we contacted them. All those residing in Yaounde, and who granted their consent were invited to the service for an interview. The date and time were determined according to the availability of the patients. In some cases, and following patient authorisation we obtained the information by phone call. Similarly, for all patients living outside Yaoundé and wishing to participate in the study, the interview was carried out on the telephone. Data on diagnosis and chemotherapy protocols were collected from the patients' medical records. For the study, we selected women following a post-chemotherapy interval of at least 12 months.

We excluded from our study all patients with a known history of hysterectomy, chemotherapy, ovarian failure or those who had a recurrence of breast cancer or died before or during our study.

Our variables of interest were socio-demographic (age, age at onset of chemotherapy, level of education, marital status), clinical (clinical stage, histological type, therapeutic modalities, chemotherapy protocol used and the number of sessions) and the characteristics of reproductive life (parity, history of infertility, contraception, menstrual cycle's pattern before and after chemotherapy, delay of menstruation after chemotherapy, desire for pregnancy, libido, occurrence of pregnancy).

We defined post-chemotherapy amenorrhoea as the absence of menses following 12 months or more of treatment cessation, and oligomenorrhoea as too few menses or bleeds lasting less than 2 days. Regular menstrual cycles are cycles occurring every 21 - 35 days. Patients were staged for breast cancer as per the TNM classification.

\begin{tabular}{lc}
\hline & Simplified AJCC TNM classification 2017 \\
\hline $\begin{array}{c}\text { Categories } \\
\text { Tumor } \\
\text { TX } \\
\text { T0 }\end{array}$ & Criteria \\
Tis & No evidence of primary tumor \\
T1 & Ductal carcinoma \\
T2 & Tumor $\leq 20$ mm in greatest dimension \\
T3 & Tumor $>20$ mm but $\leq 50$ mm in greatest dimension $>50$ mm in greatest dimension \\
T4 & Tumor of any size with direct extension to \\
T4a & Extension to the chest wall
\end{tabular}




\section{Continued}

\begin{tabular}{cc}
\hline T4b & Ulceration and/or ipsilateral macroscopic satellite nodules and/or edema of the skin \\
T4c & Both T4a and T4b \\
T4d & Inflammatory carcinoma $0.2 \mathrm{~mm}$ \\
Nodes & Regional lymph nodes cannot be assessed \\
cNX & No regional lymph node metastases \\
cN0 & Metastases to movable ipsilateral axillary lymph node (s) \\
cN1 & Metastases in ipsilateral axillary lymph nodes fixed or matted Or in \\
cN2 & ipsilateral internal mammary nodes in the absence of axillary lymph node metastases \\
Metastases & No clinical or radiographic evidence of distant metastases \\
M0 & Distant metastases detected by clinical and radiographic means \\
cM1 & Any histologically proven metastases in distant organs; \\
or if in non-regional nodes, metastases greater than 0
\end{tabular}

At the medical Oncology service of the Yaoundé General Hospital, chemotherapy is administered in cycles every 21 days. The most commonly used protocols were:

\begin{tabular}{cc}
\hline Protocols & Description \\
\hline FAC50 & $\begin{array}{r}5 \text {-fluorouracil } 500 \mathrm{mg} / \mathrm{m}^{2}+\text { Adriblastine } 50 \mathrm{mg} / \mathrm{m}^{2} \\
+ \text { Cytoxan } 500 \mathrm{mg} / \mathrm{m}^{2}(6-8 \text { cycles })\end{array}$ \\
AC60-Taxotere & $\begin{array}{r}\text { Adriamycin } 60 \mathrm{mg} / \mathrm{m}^{2}+\text { Cyclophosphamide } 600 \mathrm{mg} / \mathrm{m}^{2} \\
\text { AC60 }\end{array}$ \\
TAC & Adriamycin $60 \mathrm{mg} / \mathrm{m}^{2}+$ Cyclophosphamide $600 \mathrm{mg} / \mathrm{m}^{2}$ \\
FUN & Taxol $135 \mathrm{mg} / \mathrm{m}^{2}\left(\mathrm{or} 80 \mathrm{mg} / \mathrm{m}^{2}\right.$ if weekly administration $)$ \\
+ Adriamycin $50 \mathrm{mg} / \mathrm{m}^{2}+$ Cyclophosphamide $500 \mathrm{mg} / \mathrm{m}^{2}$
\end{tabular}

In the event of over expression of HER2, the weekly Taxotere or Navelbine regimens were proposed in association with Trastuzumab.

We analysed our data using SPSS 23.0 and Microsoft Excel 2010 software, and presented our results as means $(+/-S D)$ for quantitative variables and frequency variables for qualitative variables.

\section{Results}

Between January 2011 and December 2015, 900 cases of breast cancer were managed at the Medical oncology unit of the Yaoundé General Hospital. From amongst these, 406 patients were selected after evaluating the information contained in the database of the Medical Oncology unit and the patient's medical records. The other files were those of patients who did not start chemotherapy. From the 406 patients, 141 patients were excluded amongst which: 31 deceased 
patients, 100 who were still in chemotherapy for local or metastatic recurrence, 5 who had incomplete or absent records, 5 who had a history of hysterectomy before the diagnosis of breast cancer, and 1 who had a history of chemotherapy prior to the diagnosis of breast cancer. In the end, 265 patients were included for the study (Figure 1).

\section{Sociodemographic characteristics}

The average age of the patients at the time of the study was $39.2 \pm 6.8$ years, while the average age of patients at the onset of chemotherapy was $35.9 \pm 6.6$ years. Higher and secondary education levels were the most represented, representing $37 \%$ and $36.2 \%$ of cases respectively. Half of these were married (Table 1).

\section{Clinical and histopathological characteristics}

Table 2 represents clinical and histopathological characteristics. Pauciparous patients represented $68.3 \%(181 / 265)$ of the cases. Root canal carcinoma was the most diagnosed histological type (90.6\%/240/265). The diagnosis was often made in advanced stages of the disease, i.e. T3 and T4 in proportions of $34.3 \%$ $(96 / 265)$ and 39.2\% (98/265) respectively.

\section{Therapeutic variables}

Table 3 represents the therapeutic aspects. The most common management plan for patients involved chemotherapy, surgery and radiotherapy in $70.6 \%$ (187/265) of cases. The FAC and AC60-Taxotere protocols were the most commonly used, in $44.5 \%$ (118/265) and 35.5\% (94/265) of cases respectively.

Table 1. Patients socio demographic characteristics before CT $(\mathrm{N}=265)$.

\begin{tabular}{lcc}
\hline \multicolumn{1}{c}{ Variables } & Number $(\mathrm{n})$ & Frequency $(\%)$ \\
\hline Profession & 70 & 26.4 \\
housewife & 65 & 24.5 \\
civil servant & 57 & 21.5 \\
Trader & 53 & 20.0 \\
Private sector & 20 & 7.4 \\
Student & & \\
Educational status & 98 & 37.0 \\
Higher & 96 & 36.2 \\
Secondary & 68 & 25.7 \\
Primary & 3 & 1.1 \\
Nil & & \\
Marital Status & 135 & 51.0 \\
Married & 29 & 10.9 \\
Celibate & 87 & 32.8 \\
Free union & 6 & 2.3 \\
Divorced & 8 & 3.0 \\
Widow & & \\
\hline
\end{tabular}




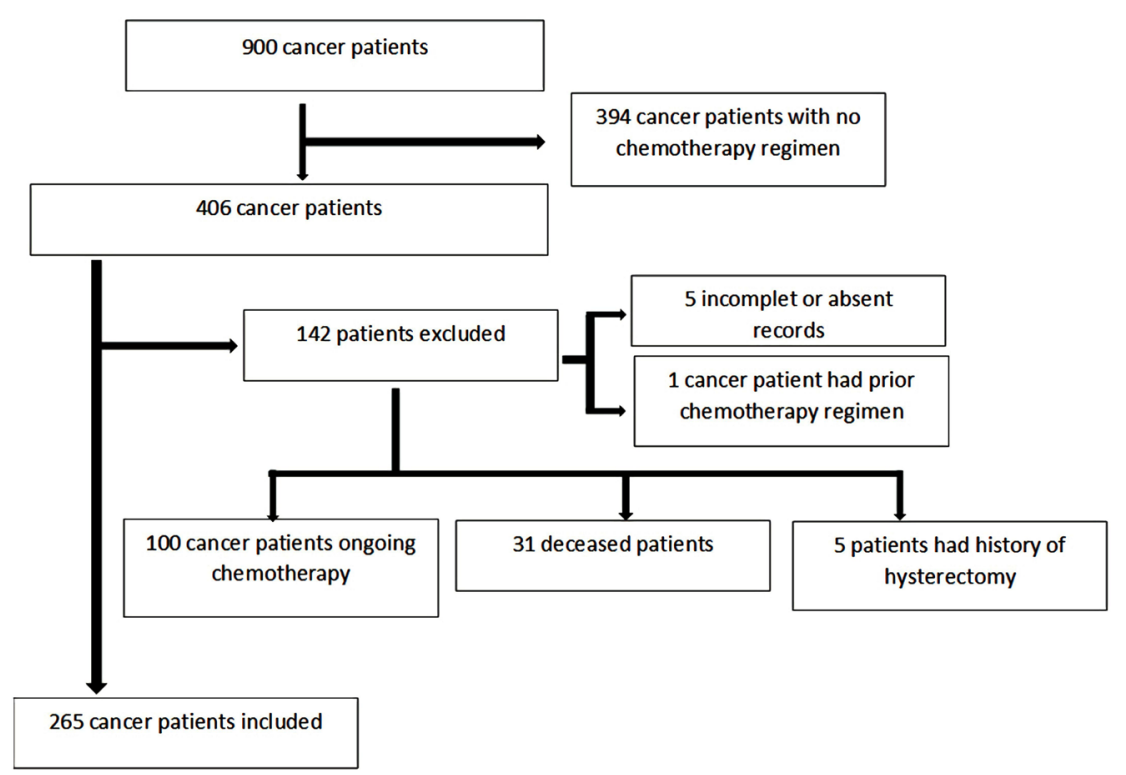

Figure 1. Flow chart of the screening process.

Table 2. Clinical and histopathological characteristics before CT $(\mathrm{N}=265)$.

\begin{tabular}{lcc}
\hline \multicolumn{1}{c}{ Variables } & Number $(\mathrm{n})$ & Frequency $(\%)$ \\
\hline Age (years) at chemotherapy onset & 28 & 10.6 \\
$<30$ & 126 & 47.5 \\
$30-39$ & 111 & 41.9 \\
$\geq 40$ & & \\
Parity & 27 & 10.2 \\
Nulliparous & 181 & 68.3 \\
Pauciparous & 57 & 21.5 \\
Multiparous & & \\
Menstrual cycle & 237 & 89.4 \\
Regular & 28 & 10.6 \\
Irregular & & \\
Histological types & 240 & 90.6 \\
Ductal carcinoma & 18 & 6.8 \\
Lobular carcinoma & 3 & 1.1 \\
Sarcoma & 1 & 0.4 \\
Paget's disease & 1 & 0.4 \\
Papillar carcinoma & 2 & 0.8 \\
Mixte carcinoma & & 3.3 \\
Clinical stage of the disease & 104 & \\
T1 & 22 & \\
T2 & & \\
T3 & & \\
T4 & & \\
TX & & \\
\hline
\end{tabular}


Table 3. Patient distribution by treatment modalities $(\mathrm{N}=265)$.

\begin{tabular}{lcc}
\hline \multicolumn{1}{c}{ Variables } & Number (n) & Frequency (\%) \\
\hline Management plan adopted & 187 & 70.6 \\
SCR & 1 & 0.4 \\
SCT & 7 & 2.6 \\
SCRT & 49 & 18.5 \\
SCRH & 15 & 5.7 \\
SC & 6 & 2.3 \\
S & & \\
Chemotherapy protocol applied & 94 & 35.5 \\
AC60-Taxotere & 118 & 44.5 \\
FAC & 2 & 0.8 \\
AC60-Taxotere-Adriamycin & 41 & 15.5 \\
AC60 & 3 & 1.1 \\
FAC-FUN & 7 & 2.6 \\
AC60-Taxotere-Herceptin & & \\
\hline
\end{tabular}

S: Surgery; SC: Surgery-Chemotherapy; SCHH: Surgery-Chemotherapy-Radiotherapy-Hormonotherapy; SCRT: Surgery-Chemotherapy-Radiotherapy-Targeted therapy; SCT: Surgery-Chemotherapy-Targeted therapy; SCR: Surgery-Chemotherapy-Radiotherapy.

\section{Reproductive life variables}

Table 4 and Table 5 represent the reproductive life characteristics of our participants. Amongst the patients included, $11.7 \%$ (31/265) claimed to have infertility prior to breast cancer diagnosis. Regarding 188 patients who experienced a return of menses, the average period of return was $6.3+/-2.0$ months, with extremes ranging from 1 to 11 months. The proportion of patients with an irregular cycle increased after chemotherapy from $10.6 \%$ (28/265) prior to $41.2 \%$ (109/265) after chemotherapy (Table 5). Seventy-seven patients (29.1\%; 77/265) had chemotherapy-induced amenorrhea (Table 5), and most of them were above 40 years (90.1\%; 70/77). Furthermore, 98/265 (37.0\%) menstruated anew after 6 months. Patients reported a decrease in libido in 51.3\% (136/265) of the cases and $13.2 \%$ (35/265) of them no longer had sexual intercourse after chemotherapy.

The majority of patients had a desire for pregnancy after chemotherapy $(71.3 \% ; 189 / 265)$ and this desire was especially expressed in the [30 - 40] year age group (Figure 2). The crude pregnancy rate 13 months after the end of chemotherapy was $6.4 \%(17 / 265)$ while the pregnancy rate was $22.4 \%(17 / 76)$ if we take into account women who expressed a desire for pregnancy. Most women who had a pregnancy had a desire to conceive $(11.8 \%, 9 / 76)$ compared to the others $(4.2 \%, 8 / 189)$ (Table 6$)$ and all age groups before 40 years were concerned (Table 7). 
Table 4. Reproductive life data of patients after chemotherapy $(\mathrm{N}=265)$.

\begin{tabular}{|c|c|c|}
\hline Variables & Number (n) & Frequency (\%) \\
\hline \multicolumn{3}{|l|}{ Menstrual cycle characteristics } \\
\hline regular & 156 & 58.9 \\
\hline oligomenorrhoea & 32 & 12.1 \\
\hline amenorrhea after 12 months & 77 & 29.1 \\
\hline \multicolumn{3}{|c|}{ Onset of menses after end of chemotherapy* } \\
\hline within 3 months & 14 & 5.3 \\
\hline within 6 months & 98 & 37.0 \\
\hline within 9 months & 180 & 67.9 \\
\hline within 12 months & 188 & 70.9 \\
\hline \multicolumn{3}{|l|}{ Libido levels } \\
\hline Preserved & 122 & 46.0 \\
\hline lowered & 136 & 51.3 \\
\hline no response & 7 & 2.6 \\
\hline \multicolumn{3}{|l|}{ Weekly coital activity (day) } \\
\hline no sexual intercourse & 35 & 13.2 \\
\hline 1 & 109 & 41.1 \\
\hline 2 & 96 & 36.2 \\
\hline 3 and more & 25 & 9.4 \\
\hline \multicolumn{3}{|l|}{ Pregnancy desire } \\
\hline Yes & 76 & 28.7 \\
\hline No & 189 & 71.3 \\
\hline \multicolumn{3}{|l|}{ Pregnancy occurrence by age } \\
\hline$<30$ & 8 & 3.0 \\
\hline $30-39$ & 8 & 3.0 \\
\hline$>40$ & 1 & 0.4 \\
\hline
\end{tabular}

*cumulative number of women.

Table 5. Menstrual cycle characteristics after chemotherapy by age range $(\mathrm{N}=265)$.

\begin{tabular}{ccccc}
\hline Menstrual cycle & $\mathbf{2 0 - 2 9}$ & $\mathbf{3 0 - 3 9}$ & $\geq 40$ & Total \\
\hline Normal & $28(17.9)$ & $105(67.3)$ & $23(14.7)$ & $156(58.9)$ \\
Oligomenorrhea & 0 & $14(43.8)$ & $18(56.2)$ & $32(12.1)$ \\
Amenorrhoea & 0 & $7(9.1)$ & $70(90.9)$ & $77(29)$ \\
\hline
\end{tabular}

Table 6. Distribution of pregnancy occurrence by age.

\begin{tabular}{ccccc}
\hline Variables & {$[20-30]$} & ] $30-40]$ & ] $40-49]$ & Total \\
\hline Pregnancy & $8(28.6)$ & $8(6.3)$ & $1(0.9)$ & $17(6.4)$ \\
No pregnancy & $20(71.4)$ & $118(93.7)$ & $110(99.1)$ & $248(94.6)$ \\
Total & $28(100)$ & $126(100)$ & $111(100)$ & $265(100)$ \\
\hline
\end{tabular}


Table 7. Distribution of pregnancy occurrence according to pregnancy desire.

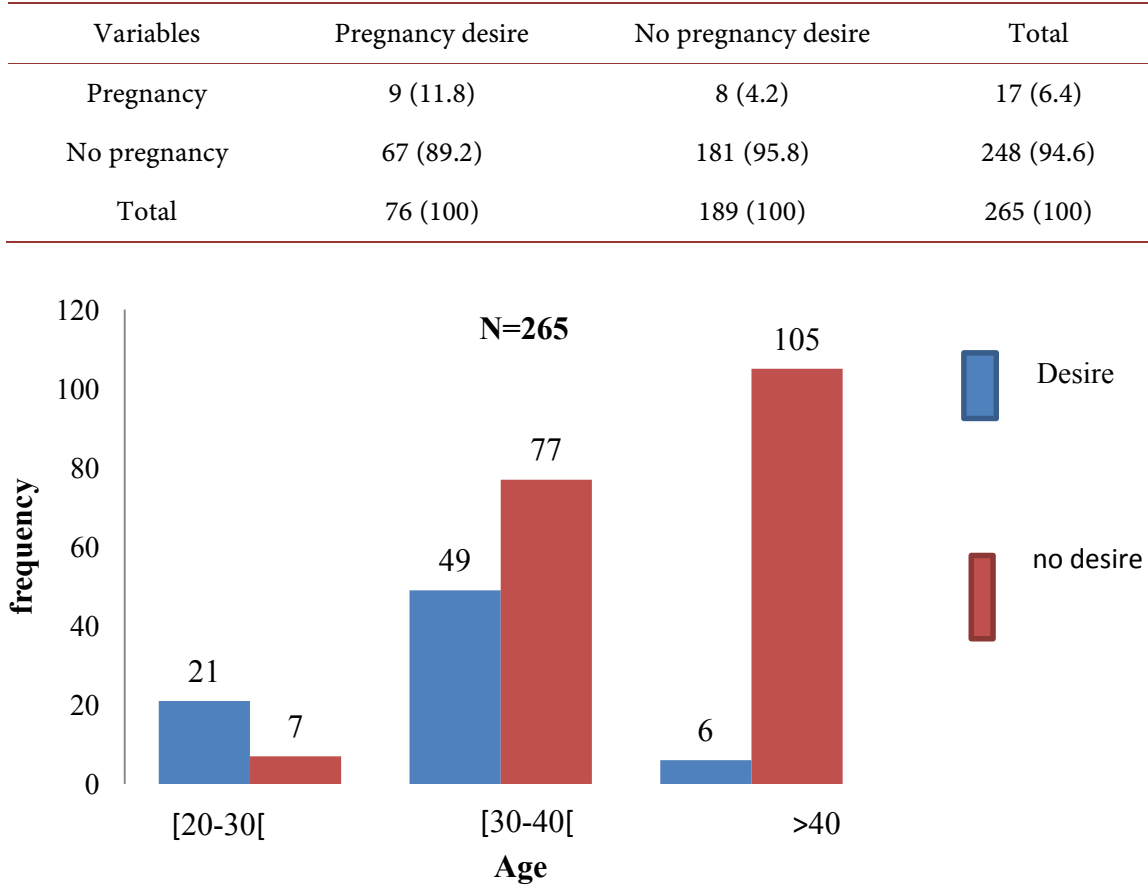

Figure 2. Distribution of pregnancy desire by age range $(\mathrm{N}=265)$.

\section{Discussion}

The occurrence of amenorrhea following chemotherapy reveals its toxicity to the ovaries. Several authors have echoed this in many studies [10] [11] [12] [13] [14]. The possibility of premature ovarian failure caused by chemotherapy, is potentially invalidating for women of childbearing age treated for breast cancer. According to Kemfang et al. (2011) [3] about 30\% of breast cancers diagnosed in Cameroon affect women under 40 years of age. Most of the women in our study population who desired pregnancy (28.1\%) were between 31 and 40 years of age.

Despite their young age, the majority of our patients (73.5\%) had locally advanced-stage disease (T3, T4) at the time of diagnosis, which is frequently the case in resource-limited countries [2] [3] [5] [7]. This is because patients generally seek medical attention late in the course of disease [1] [12], unlike in industrialized countries where the diagnosis is made earlier [13]. The late discovery of the disease is associated with poor survival rates [2], ultimately leading to aggressive treatments, especially in young women [14]. These treatments are likely to be more toxic to the ovaries.

In addition, young age is a poor prognostic factor [15] for breast cancer. The average age in our study population was 35.9 years.

Many authors have evaluated the toxicity of the chemotherapeutic agents used in the management of breast cancers, with evidence that treatment protocols affect ovarian function differently. The toxicity depends on the age of the patient, the agent used, including the dose and the protocol [16]. Cyclophosphamide-based protocols induce the highest rates of chemotherapy-induced ovarian 
failure especially after 40 years [16] [17]. Long JP et al. [18] had found that although both CAF and DPT affected ovarian function, alterations with the CAF protocol was less pronounced. The FAC protocol (44.5\%) was the most used in our study, a fact not consistent with recent literature, which increasingly recommends a sequence-based protocol with Anthracyclines and Taxanes, known to be less toxic to ovaries [19] [20]. The reason for this is that many chemotherapeutic agents remain unavailable in our setting, and their relatively high cost makes them inaccessible for the majority of these patients who have limited resources in the absence of a Universal health coverage scheme.

Age is a determining factor in the occurrence of menstrual disorders following chemotherapy. The average age of women in our study was young at 35.9 years. This is probably why only $41.1 \%$ of our patients had cycle disorders such as amenorrhoea or oligomenorrhoea after chemotherapy. In the study by Tiong et al. [21], 93.1\% of women reported amenorrhea at the end of treatment and $77.9 \%$ reported same 12 months after chemotherapy in a female population in which the average age was 43.3 years. These authors also found that age was the most important risk factor for the occurrence of chemotherapy-induced amenorrhoea because the rate of amenorrhoea increased with increasing age. This finding was further corroborated by the work of Ben-Aharon et al. [22], which showed that women under 35 years of age recovered from alterations in chemotherapy-induced ovarian circulation faster than their older counterparts. Menstrual disorders are likely to occur in women who already have some degree of aging ovarian function [23] [24]. Amongst the women who complained of amenorrhea, $37 \%$ had a resumption of menstrual activity after 6 months. This rate was higher than those described in literature with different treatment protocols [21].

We observed $17(6.4 \%)$ cases of spontaneous pregnancies 13 months after chemotherapy, and a $10.4 \%$ pregnancy rate among women under 40 years of age. Despite the return to normal of the menstrual cycle, young women survivors of chemotherapy will undergo varying degrees of altered ovarian function that will interfere with their fertility [25].

The pregnancy rate amongst women over 40 years of age was extremely low at $0.9 \%$ with Gerber B et al. [26] reporting a 3\% spontaneous pregnancy rate amongst women over 40 years of age, suggesting a more severe alteration in fertility in women over 40 years of age, even if their menstrual cycle remains intact.

Literature findings estimate that $70 \%$ of patients under 45 years of age who are treated for breast cancer would desire a child after the end of their treatment [27], however we found a lower proportion in our study. This could be explained by the fact that most of our patients already had at least one child. In order to avoid the residual teratogenic risk of chemotherapy, a minimum of at least 1 year following the last course of chemotherapy is recommended (at least 3 months after stopping Tamoxifen) [28]. Unfortunately safety time is not often taken into consideration.

Our study presented some limits. First, we studied overall female fertility fol- 
lowing chemotherapy regardless of the protocol used. Also, the size of our sample did not allow us to isolate the protocol favorable to fertility, or better still to identify the molecule most toxic to the ovaries. In addition, data obtained by telephone posed the risk of information bias. Finally, we evaluated the fertility of women and not that of the couple, with the risk of missing potential male partner infertility as the sole cause for childlessness.

\section{Conclusion}

Chemotherapy alters the fertility of women treated for breast cancer. Cycle disorders are more frequent after chemotherapy. The resumption of normal menstrual cycle activity does not guarantee future childbearing. The high proportions of patients wanting pregnancy after chemotherapy suggest the need to integrate fertility consultations into the management of breast cancer patients and to look out for factors associated with fertility potential following chemotherapy.

\section{Acknowledgements}

The authors thank the administrative personnel of the Yaoundé General Hospital for facilitating the implementation of this study as well as staff of the service and personnel of the oncology service for their support during the data collection. Apart from the personal contribution of each author, the study was not funded.

\section{Data Availability Statement}

The data used to support the findings of this study are included within the article.

\section{Funding Statement}

This article was not funded.

\section{Acknowledgements}

Avoid the stilted expression, "One of us (R. B. G.) thanks..." Instead, try "R. B. G. thanks". Do NOT put sponsor acknowledgements in the unnumbered footnote on the first page, but at here.

\section{Conflicts of Interest}

The authors declare that there are no conflicts of interest regarding the publication of this paper.

\section{References}

[1] Enow, O.G.E., Ndom, P. and Doh, A.S. (2012) Current Cancer Incidences and Trends in Yaounde, Cameroon. Oncology, Gastroenterology and Hepatology Reports, 1, 58-63. https://doi.org/10.4103/2348-3113.133639

[2] Essiben, F., Foumane, P., Meka, E.J.N.U., Tchakounté, M., Dohbit, S.J., Nsahlai, C., 
et al. (2017) Descriptive Analysis of 192 Cases of Breast Cancer Occurring before Age 40 in Yaounde, Cameroon. International Journal of Reproduction, Contraception, Obstetrics and Gynecology, 6, 2704-2710. https://doi.org/10.18203/2320-1770.ijrcog20172898

[3] Kemfang, N.J.D., Yomi, J., Kasia, J.M., Mawamba, Y., Ekortarh, A.C. and Vlastos, G. (2011) Breast Cancer Profile in a Group of Patients Followed up at the Radiation Therapy Unit of the Yaounde General Hospital, Cameroon. Obstetrics and Gynecology International, 2011, Article ID: 143506. https://doi.org/10.1155/2011/143506

[4] Lee, M.K., Varzi, L.A., Chung, D.U., Cao, M., Gornbein, J., Apple, S.K., et al. (2015) The Effect of Young Age in Hormone Receptor Positive Breast Cancer. BioMed Research International, 2015, Article ID: 325715. https://doi.org/10.1155/2015/325715

[5] Bano, R., Salim, M., Abid, M., Akif Zaidi and Khan, A.I. (2016) Prognosis of Breast Cancer in Very Young Age (Less than 30 Years). Journal of Cancer \& Allied Specialties, 2, 4. https://doi.org/10.37029/jcas.v2i1.53

[6] Mazeh, H., Sagiv, I., Katz, D., Freund, H.R., Peretz, T. and Allweis, T.M. (2013) Association between Patient Age, Volume of Breast Tissue Excised, and Local Recurrence. Journal of Surgical Research, 181, 187-192.

https://doi.org/10.1016/j.jss.2012.06.043

[7] Sando, Z., Fouogue, J.T., Fouelifack, F.Y., Fouedjio, J.H., Mboudou, E.T. and Essame, J.L.O. (2014) Profil des cancers gynécologiques et mammaires à Yaoundé-Cameroun. Pan African Medical Journal, 17, 28. https://doi.org/10.11604/pamj.2014.17.28.3447

[8] Rudra, S., Yu, D.S., Yu, E.S., Switchenko, J.M., Mister, D. and Torres, M.A. (2015) Locoregional and Distant Recurrence Patterns in Young versus Elderly Women Treated for Breast Cancer. International Journal of Breast Cancer, 2015, Article ID: 213123. https://doi.org/10.1155/2015/213123

[9] Morgan, S., Anderson, R.A., Gourley, C., Wallace, W.H. and Spears, N. (2012) How Do Chemotherapeutic Agents Damage the Ovary? Human Reproduction Update, 18, 525-535. https://doi.org/10.1093/humupd/dms022

[10] Meirow, D., Dor, J., Kaufman, B., Shrim, A., Rabinovici, J., Schiff, E., et al. (2007) Cortical Fibrosis and Blood-Vessels Damage in Human Ovaries Exposed to Chemotherapy. Potential Mechanisms of Ovarian Injury. Human Reproduction, 22, 1626-1633. https://doi.org/10.1093/humrep/dem027

[11] Woodruff, T.K. (2010) The Oncofertility Consortium-Addressing Fertility in Young People with Cancer. Nature Reviews Clinical Oncology, 7, 466-475. https://doi.org/10.1038/nrclinonc.2010.81

[12] Ngowa, J., Kasia, J., Yomi, J., Nana, A., Ngassam, A., Domkam, I., et al. (2015) Breast Cancer Survival in Cameroon: Analysis of a Cohort of 404 Patients at the Yaoundé General Hospital. Advances in Breast Cancer Research, 4, 44-52. https://doi.org/10.4236/abcr.2015.42005

[13] Azim, A.A., Costantini-Ferrando, M. and Oktay, K. (2008) Safety of Fertility Preservation by Ovarian Stimulation with Letrozole and Gonadotropins in Patients with Breast Cancer: A Prospective Controlled Study. Journal of Clinical Oncology, 26, 2630-2635. https://doi.org/10.1200/JCO.2007.14.8700

[14] Slaoui, M., Mouh, F.Z., Ghanname, I., Razine, R., El Mzibri, M. and Amrani, M. (2016) Outcome of Breast Cancer in Moroccan Young Women Correlated to Clinic-Pathological Features, Risk Factors and Treatment: A Comparative Study of 716 Cases in a Single Institution. PLOS ONE, 11, e0164841. https://doi.org/10.1371/journal.pone.0164841 
[15] Chollet-Hinton, L., Anders, C.K., Tse, C.K., Bell, M.B., Yang, Y.C., Carey, L.A., et al. (2016) Breast Cancer Biologic and Etiologic Heterogeneity by Young Age and Menopausal Status in the Carolina Breast Cancer Study: A Case-Control Study. Breast Cancer Research, 18, 79. https://doi.org/10.1186/s13058-016-0736-y

[16] Mathelin, C., Brettes, J.P. and Diemunsch, P. (2008) Premature Ovarian Failure after Chemotherapy for Breast Cancer. Bulletin du Cancer, 95, 403-412.

[17] Oktem, O. and Oktay, K. (2007) Quantitative Assessment of the Impact of Chemotherapy on Ovarian Follicle Reserve and Stromal Function. Cancer, 110, 2222-2229. https://doi.org/10.1002/cncr.23071

[18] Long, J.P., Wan, F., Zhang, F., Zhou, J. and Don, L.F. (2016) DTC Chemotherapy Regimen Is Associated with Higher Incidence of Premature Ovarian Failure in Women of Reproductive Age with Breast Cancer. European Review for Medical and Pharmacological Sciences, 20, 1087-1092.

[19] Cardoso, F., Loibl, S., Pagani, O., Graziotin, A., Panizza, P., Martincich, L., et al. (2012) The European Society of Breast Cancer Specialists Recommendations for the Management of Young Women with Breast Cancer. European Journal of Cancer, 48, 3355-3377. https://doi.org/10.1016/j.ejca.2012.10.004

[20] Mailliez, A., Decanter, C. and Bonneterre, J. (2011) Adjuvant Chemotherapy for Breast Cancer and Fertility: Estimation of the Impact, Options of Preservation and Role of the Oncologist. Bulletin du Cancer, 13, 75-84.

[21] Tiong, V., Rozita, A.M., Taib, N.A., Yip, C.H. and Ng, C.H. (2014) Incidence of Chemotherapy-Induced Ovarian Failure in Premenopausal Women Undergoing Chemotherapy for Breast Cancer. World Journal of Surgery, 38, 2288-2296. https://doi.org/10.1007/s00268-014-2542-y

[22] Ben-Aharon, I., Granot, T., Meizner, I., Hasky, N., Tobar, A., Rizel, S., et al. (2015) Long-Term Follow-Up of Chemotherapy-Induced Ovarian Failure in Young Breast Cancer Patients: The Role of Vascular Toxicity. Oncologist, 20, 985-991. https://doi.org/10.1634/theoncologist.2015-0044

[23] Berliere, M., Dalenc, F., Malingret, N., Vindevogel, A., Piette, P., Roche, H., et al. (2008) Incidence of Reversible Amenorrhea in Women with Breast Cancer Undergoing Adjuvant Anthracycline-Based Chemotherapy with or without Docetaxel. BMC Cancer, 8, 56. https://doi.org/10.1186/1471-2407-8-56

[24] Hortobagyi, G., Buzdar, A.U., Marcus, C.E. and Smith, T.L. (1986) Immediate and Long-Term Toxicity of Adjuvant Chemotherapy Regimens Containing Doxorubicin in Trials at M.D. Anderson Hospital and Tumor Institute. NCI Monographs, 1, 105-109.

[25] Morarji, K., McArdle, O., Hui, K., Gingras-Hill, G., Ahmed, S., Greenblatt, E.M., et al. (2017) Ovarian Function after Chemotherapy in Young Breast Cancer Survivors. Current Oncology, 24, e494-e502. https://doi.org/10.3747/co.24.3335

[26] Gerber, B., Dieterich, M., Muller, H. and Reimer, T. (2008) Controversies in Preservation of Ovary Function and Fertility in Patients with Breast Cancer. Breast Cancer Research, Treat, 108, 1-7. https://doi.org/10.1007/s10549-007-9572-1

[27] Partridge, A.H. and Ruddy, K.J. (2015) Fertility and Adjuvant Treatment in Young Women with Breast Cancer. Breast, 16, S175-S181. https://doi.org/10.1016/j.breast.2007.07.029

[28] This, P. (2008) Breast Cancer and Fertility: Critical Review, Considerations and Perspectives. Bulletin du Cancer, 95, 17-25. 